\title{
A New Method to Build Covariance of Gravity Anomaly Along with its Simulation Experience
}

\author{
Jianbo Wang*,1,2 jinyun Guo $^{1,2}$ and Youge Xie $\mathrm{X}^{1,2}$ \\ ${ }^{1}$ College of Geodesy and Geomatics, Shandong University of Science and Technology, Qingdao 266590, China \\ ${ }^{2}$ Key Laboratory of Surveying and Mapping Technology on Island and Reef of NASMG, Qingdao 266590, China
}

\begin{abstract}
In this paper, we proposed a new method to build covariance matrix of gravity anomaly based on the spherical harmonic expansion of gravity anomaly, and then combined with the measured gravity data we can realize the fast and high precision interpolation and extrapolation of gravity anomaly according to the principle of least squares and the remove-compute-restore technique. The feasibility of this method is proved through the simulation experiment.
\end{abstract}

Keywords: Covariance matrix of gravity anomaly, least squares collocation, the remove-compute-restore technique.

\section{INTRODUCTION}

Covariance function has been becoming a basic content of physical geodesy research since the discover of the least squares collocation theory. Many important problems in physical geodesy utilize integration calculation which are to be extended to the global, so it is needed to measure the gravity value of every point in the earth surface for solving this problem in theory. In fact, there are only few relative points measurement even in the most dense gravity network and in most of the blank areas the gravity estimation is needed. According to the principle of least squares collocation [1-4], the unknown signal can computed using the value of the observation, the covariance of observation and the cross-covariance of observation and signal. The unknown signal can be similar kind with the observation also can be different kind of it. So we can conclude that we can calculate high-accuracy other gravimetric values e.g. deflection of vertical and undulation of geoid using highaccuracy of gravity of few measured points .This point is very important because the gravity measurement is more easier than other gravimetric value measure e.g. deflection of vertical and undulation of geoid in some areas such as in mountains and oceans. And the crucial issue is how to build covariance matrix accurately which is the key to get high precision unknown signal. In previous studies, many scholars home and abroad gives the empirical approximation formula $[5,6]$ based on the statistical approximation method, but this approximation formula is not applicable widely , because the local gravity field of the earth is different in different areas and the changes of it is complex in many areas, and it is impossible to calculate the accurate covariance function of gravity anomaly just through a small amount of gravity values in the sparse distribution of the local area. For that reason, the application of this theory is not widely used in practice. Least squares collocation is an excellent prediction method, especially in the application of interpolation and extrapolation of gravity anomaly, people mainly focus on the local statistical approximation of covariance functions, but these are not widely used functions, because the local gravity anomaly covariance functions related to the local terrain of the research areas, the distribution and density of the local gravity observation. The function of approximation formula derived from an research area cannot be used in other areas. Some scholars [7] provide a new method using terrain height covariance function to approximate the gravity anomaly covariance function on the basis of the idea that there are strong correlation between the free-air gravity anomaly and topography. This method directly describe the characteristics of gravity anomaly linear correlation associated with the elevation in nature, and at the same time, this method provide a new idea of application of high resolution digital terrain model to alleviate the workload in gravity observation. But the application of this method is still limited because of the heavy workload and high cost due to the diversity of experience formula functions and we must retested before each application for the different parameters of covariance functions in different terrains.

With the development of science and new technology, the method of gravity measurement is become more and more diversified, mainly including land gravity survey, marine gravity measurement, aviation or airborne gravity measurement, satellite gravity measurement which is including the ground track of satellite orbit perturbation, satellite altimetry, satellite to satellite tracking and satellite gradiometry. The application of these new techniques are taking the study of the earth's gravitational model to a new level. EGM2008 [8] which is the world's ultra-high degree of earth gravitational model released by the earth's gravity field research and development team of the National Geospatial Intelligence Agency (NGA) of USA, the total order of EGM2008 up to 2159 (extended to 2190 order in the 
spherical harmonic coefficients), the model spatial resolution is equivalent to about 5 minute (about $9 \mathrm{~km}$ ). The root mean square of geoid globally achieve the accuracy of $15 \mathrm{~cm}$.

This global set of gravity anomalies was formed by merging terrestrial and airborne data with altimetry-derived values, the rate of data acquisition for ground surface reached $83 \%$ and other area where the available gravity anomaly data could only be used at a lower resolution ,mainly in the South Pole, their spectral content was supplemented with gravitational information obtained from airborne data with altimetry-derived values.EGM2008 was developed in a least squares adjustment that combined the ITG-GRACE03S model with the ITG-GRACE03S coefficient and the association covariance information which is the main source of the low degree of EGM2008 gravitational coefficient and the precision of EGM2008 is very high [9]. Over areas covered with high quality gravity data (e.g., USA, Europe, Australia), the discrepancies between geoid undulations computed from EGM2008 and those computed from independent GPS/Leveling data are on the order of 5 to $10 \mathrm{~cm}$. These results are comparable to, and in several cases better than, corresponding results obtained using regional detailed geoid models [8].

In this paper, the aim is to propose a new method to build the covariance matrix of anomaly based on the spherical harmonic expansion of gravity anomaly , and combined with the measured gravity data then we can realize the fast and high precision interpolation and extrapolation of gravity anomaly according to the principle of least squares and the remove-compute-restore technique [10]. With the development of the ultra-high degree gravitational model, it will becomes a convenience way to build the high precision gravity anomaly covariance matrix.

\section{THE GRAVITY ANOMALY COVARIANCE MATRIX OF SPHERICAL HARMONIC EXPANSION}

\subsection{The Formula of Spherical Harmonic Expansion of Gravity Anomaly}

The earth's gravitational model theoretically can be seen as integer order and degree of spherical harmonic which is convergent or infinite series of spherical harmonics function. The series of the gravitational potential of the earth can be written as $[8,5,11]$.

$V(r, \theta, \lambda)=\frac{G M}{r}\left[1+\sum_{n=2}^{N \max }\left(\frac{a}{r}\right)^{n} \sum_{m=0}^{n}\left(\bar{C}_{n m} \cos m \lambda+\bar{S}_{n m} \sin m \lambda\right) \bar{P}_{n m}(\cos \theta)\right](1)$

where $G M$ is the geocentric gravitational constant $3986004.415 \times 10^{8} \mathrm{~m}^{3} \mathrm{~s}^{-2}$ and $a$ is equal to $6378136.3 \mathrm{~m}$ meaning the semi-major-axis of normal ellipsoid and $r, \theta, \lambda$ denote the spherical coordinates of the interesting point. The $\bar{C}_{n m}, \bar{S}_{n m}$ is the fully normalized spherical harmonic coefficients and $\bar{P}_{n m}(\cos \theta)$ is the fully normalized Legendre association function of the first kind of degree $n$ and order $m$. The maximum degree $N_{\max }$ that is commensurate with the size of the equi-angular cells forming the global grid.

The spherical harmonic expansion of the normal gravitational potential is given by:
$V^{\prime}(r, \theta, \lambda)=\frac{G M}{r}\left[1+\sum_{n=1}^{N \max }\left(\frac{a}{r}\right)^{2 n} \bar{C}_{2 n}^{\prime} \bar{P}_{2 n}(\cos \theta)\right]$.

The disturbing potential $T$ is describe as,

$T=W-U=V-V^{\prime}$

where $W$ is the gravitational potential of earth and $U$ is the normal gravitational potential. The spherical harmonic expansion of the disturbing potential $T$ [12] is given by:

$T(r, \theta, \lambda)=\frac{G M}{r} \sum_{n=1}^{\infty}\left(\frac{a}{r}\right)^{n} \sum_{m=0}^{N \max }\left(\bar{C}_{n m}^{*} \cos m \lambda+\bar{S}_{n m} \sin m \lambda\right) \bar{P}_{n m}(\cos \theta)$

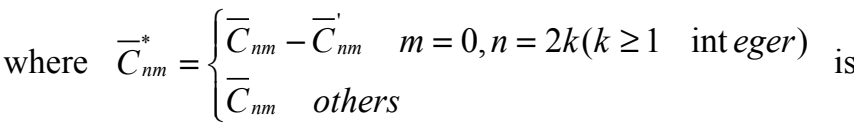
the coefficient of potential that are normal gravitational potential subtracted from gravitational potential.

We all known that the Bruns formula is written as,

$$
\Delta g=-\frac{\partial T}{\partial r}-\frac{2}{r} T
$$

so that, from equation (3), we have:

$$
\Delta g=\frac{G M}{r^{2}} \sum_{n=1}^{N \max }(n-1)\left(\frac{a}{r}\right)^{n} \sum_{m=0}^{n}\left(\bar{C}_{n m}^{*} \cos m \lambda+\bar{S}_{n m} \sin m \lambda\right) \bar{P}_{n m}(\cos \theta)
$$

\section{2. the Building of Covariance of Gravity Anomaly}

Translate the formula (6) into the following format,

$$
\begin{gathered}
\Delta g=\frac{G M}{r^{2}}\left\{\sum_{n=2}^{N \max } \sum_{m=0}^{n}\left[(n-1)\left(\frac{a}{r}\right)^{n} \cos m \lambda \cdot \bar{P}_{n m}(\cos \theta) \cdot \bar{C}_{n m}^{*}\right]+\right. \\
\left.\sum_{n=2}^{N \max } \sum_{m=0}^{n}\left[(n-1)\left(\frac{a}{r}\right)^{n} \sin m \lambda \cdot \bar{P}_{n m}(\cos \theta) \cdot \bar{S}_{n m}\right]\right\}
\end{gathered}
$$

with the alternative $(n-1)\left(\frac{a}{r}\right)^{n} \cos m \lambda \cdot P_{n m}(\cos \theta)=A_{n m}$ and $(n-1)\left(\frac{a}{r}\right)^{n} \sin m \lambda \cdot \bar{P}_{n m}(\cos \theta)=B_{n m}$ for the formula (7), we have,

$$
\Delta g=\frac{G M}{r^{2}}\left\{\sum_{n=2}^{N \max } \sum_{m=0}^{n}\left[A_{n m} \cdot \bar{C}_{n m}^{*}\right]+\sum_{n=2}^{N \max } \sum_{m=0}^{n}\left[B_{n m} \cdot \bar{S}_{n m}\right]\right\}
$$

suppose we have two points on the surface of earth, and the gravity anomaly $\Delta g^{P}, \Delta g^{Q}$ are respectively,

$$
\begin{aligned}
& \Delta g^{P}=\frac{G M}{r_{P}^{2}}\left\{\sum_{n=2}^{N \max } \sum_{m=0}^{n}\left[A_{n m}^{P} \cdot \bar{C}_{n m}^{*}\right]+\sum_{n=2}^{N \max } \sum_{m=0}^{n}\left[B_{n m}^{P} \cdot \bar{S}_{n m}\right]\right\} \\
& \Delta g^{Q}=\frac{G M}{r_{P}^{2}}\left\{\sum_{n=2}^{N \max } \sum_{m=0}^{n}\left[A_{n m}^{Q} \cdot \bar{C}_{n m}^{*}\right]+\sum_{n=2}^{N \max } \sum_{m=0}^{n}\left[B_{n m}^{Q} \cdot \bar{S}_{n m}\right]\right\}
\end{aligned}
$$

with the standard deviation of gravitational potential coefficient of $\sigma_{\bar{C}_{n m}}$ and $\sigma_{\bar{S}_{n m}}$ we can get the covariance matrix between the two points of $\mathrm{P}$ and $\mathrm{Q}$ according to the theory of law of error propagation, 
$\operatorname{COV}\left(\Delta g^{P}, \Delta g^{Q}\right)=\frac{G M}{r_{P}^{2}} \frac{G M}{r_{Q}^{2}}\left\{\sum_{n=2}^{N \max } \sum_{m=0}^{n}\left[A_{n m}^{P} \cdot \sigma_{\bar{c}_{m m}}^{2} \cdot A_{n m}^{Q}\right]+\sum_{n=2}^{N \max } \sum_{m=0}^{n}\left[B_{n n}^{P} \cdot \sigma_{\bar{S}_{m m}}^{2} B_{n m}^{Q}\right]\right\}$

the covariance matrix will be the variance matrix when the distance of the two points equal zero.

\section{3. the Building of Covariance of Residual Gravity Anomaly}

Remove-compute-restore theory first proposed by Forsberg \& Tscherning[13] for the purpose of improvement of the calculation accuracy. First remove the the high frequency components of the signals and makes it changed more smooth and easy interpolation, second deal with the corresponding processing data to estimation and the result add the removed high frequency components, then we get the final result[14]. According to this theory, we separated the gravity anomaly into model gravity anomaly and residual gravity anomaly, and the formula as follow,

$\Delta g=\Delta g_{G M}+\Delta g_{\text {res }}$

where $\Delta g_{\text {res }}$ is the residual gravity anomaly and $\Delta g_{G M}$ is model gravity anomaly. So from formula (11), the two points $P$ and $Q$ can written as,

$\Delta g^{P}=\Delta g_{G M}^{P}+\Delta g_{\text {res }}^{P}$

$\Delta g^{Q}=\Delta g_{G M}^{Q}+\Delta g_{\text {res }}^{Q}$

and the residual of the two points can be written as,

$\Delta g_{\text {res }}^{P}=\Delta g^{P}-\Delta g_{G M}^{P}$

$\Delta g_{\text {res }}^{Q}=\Delta g^{Q}-\Delta g_{G M}^{Q}$

according to the law of error propagation we have the residual covariance of gravity anomaly of the two points:

$\operatorname{cov}\left(\Delta g_{\text {res }}^{P}, \Delta g_{\text {res }}^{Q}\right) \approx \operatorname{cov}\left(\Delta g^{P}, \Delta g^{Q}\right)+\operatorname{cov}\left(\Delta g_{G M}^{P}, \Delta g_{G M}^{Q}\right)$

model gravity anomaly covariance may take place of the gravity anomaly covariance for approximation, then the covariance formula of residual gravity anomaly of $\mathrm{P}$ and $\mathrm{Q}$ can be written as,

$$
\begin{aligned}
& \operatorname{cov}\left(\Delta g_{\text {res }}^{P}, \Delta g_{\text {res }}^{Q}\right) \approx 2 \operatorname{cov}\left(\Delta g_{G M}^{P}, \Delta g_{G M}^{Q}\right) \\
& \quad=2\left(\frac{G M}{r_{P} r_{Q}}\right)^{2}\left\{\sum_{n=2}^{N \max } \sum_{m=0}^{n}\left[A_{n m}^{P} \cdot \sigma_{\bar{C}_{n m}^{*}}^{2} \cdot A_{n m}^{Q}\right]+\sum_{n=2}^{N \max } \sum_{m=0}^{n}\left[B_{n m}^{P} \cdot \sigma_{\bar{S}_{m m}^{2}}^{2} \cdot B_{n m}^{Q}\right]\right\}
\end{aligned}
$$

\section{LEAST SQUARE COLLOCATION ESTIMATE THE GRAVITY ANOMALY OF UNKONWN POINTS}

According to the principle of least squares collocation, the interpolation or extrapolation of unknown gravity anomaly can computed using the value of the signal gravity anomaly, the covariance of signal and the cross-covariance of unknown value and signal. The basic formula is written as,

$$
S=C_{s l}\left(C_{l l}+D\right)^{-1} L
$$

where $S$ is the interpolation or extrapolation of unknown value and $C_{s l}$ is the cross-covariance of unknown value and signal and $C_{l l}$ is the covariance of signal and $D$ is the covariance matrix of signal noise and $L$ is the signal series.

Suppose that there are series gravity anomaly signal $\Delta g_{l}$, $\Delta g_{2}, \ldots, \Delta g_{N}$,we can get the estimation formula of $P$ point for gravity anomaly from formula (18) as follow,

$$
\Delta g_{p}=\left[\begin{array}{ll}
C_{P 1}, C_{P 1}, \mathrm{~L} & C_{P N}
\end{array}\right]\left(\left[\begin{array}{cccc}
C_{11} & C_{12} & \mathrm{~K} & C_{1 N} \\
C_{21} & C_{22} & \mathrm{~K} & C_{2 N} \\
C_{31} & C_{32} & \mathrm{~K} & C_{3 N} \\
\mathrm{~K} & \mathrm{~K} & \mathrm{~K} & \mathrm{~K} \\
C_{N 1} & C_{N 2} & \mathrm{~K} & C_{N N}
\end{array}\right]+D\right)^{-1}\left[\begin{array}{c}
\Delta g_{1} \\
\Delta g_{2} \\
\mathrm{~L} \\
\Delta g_{N}
\end{array}\right]
$$

and according to the remove-compute-restore technique we can decompose the gravity anomaly series $\Delta g_{1}, \Delta g_{2}, \ldots, \Delta g_{N}$ as,

$$
\begin{array}{ll}
\Delta g=\Delta g_{G M}^{i}+\Delta g_{\text {res }}^{i} & (i=1,2, \mathrm{~L}, N) \\
\Delta g_{\text {res }}^{i}=\Delta g-\Delta g_{G M}^{i} & (i=1,2, \mathrm{~L}, N)
\end{array}
$$

and then we can estimate the residual gravity anomaly of unknown value according to the residual signal gravity anomaly as follow,

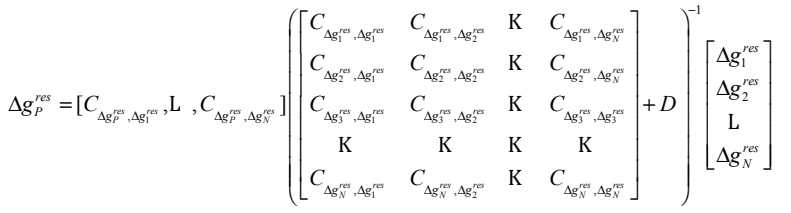

the solution $\Delta g_{p}^{\text {res }}$ add the model gravity anomaly $\Delta g_{p}{ }^{G M}$ ,then we can restore the final result of gravity anomaly of unknown point from formula (11).

Usually we suppose that there is no influence by ignoring the noise variance because it is complex to compute. But the impact of the noise covariance is also very import. So in this paper, we simulate the noise covariance using covariance of free-air gravity anomaly for the reason that there are close correlation between gravity anomaly and elevation, the formula of free-air gravity anomaly is as follows,

$$
\Delta_{1} g \approx 0.3086 H
$$

so we can get the covariance of free-air gravity anomaly according to the law of error propagation as,

$$
\operatorname{cov}\left(\Delta_{1} g^{i}, \Delta_{1} g^{i}\right)=\left[\begin{array}{cccc}
0.3086^{2} \sigma_{H_{1}}^{2} & 0.3086^{2} \sigma_{H_{1}} \sigma_{H_{2}} & \mathrm{~L} & 0.3086^{2} \sigma_{H_{1}} \sigma_{H_{N}} \\
0.3086^{2} \sigma_{H_{2}} \sigma_{H_{1}} & 0.3086^{2} \sigma_{H_{2}}^{2} & \mathrm{~L} & 0.3086^{2} \sigma_{H_{2}} \sigma_{H_{N}} \\
\mathrm{~L} & \mathrm{~L} & \mathrm{~L} & \mathrm{~L} \\
0.3086^{2} \sigma_{H_{N}} \sigma_{H_{1}} & 0.3086^{2} \sigma_{H_{N}} \sigma_{H_{2}} & \mathrm{~L} & 0.3086^{2} \sigma_{H_{N}}^{2}
\end{array}\right] \quad(i=1,2, \mathrm{~L}, N)
$$




\section{SIMULATION EXPERIENCE}

\subsection{Experience Process}

In this paper, we choose the geographic range E105. $1^{\circ}$ $\mathrm{N} 26.8^{\circ} \sim \mathrm{E} 109.3^{\circ} \mathrm{N} 30.1^{\circ}$. First download the data of SRTM3 from the CGIAR-CSI web site (http://srtm.csi.cgiar.org/SELECTION/inputCoord.asp)

shown in Fig. (1). The triangle structure net of gravity observation shown as Fig. (2), there are three given point of $1,2,3$, and a unknown point $P$. We divide the experiment into two group, for the first group with the each given side length of observation signal is about $15 \mathrm{~km}$, and the second group of each given side length of observation signal is about $30 \mathrm{~km}$. Because the elevation range for the experimental area is $361.467 \mathrm{~m} \sim 1886.489 \mathrm{~m}$, so we can divided this area into three kinds of terrain ,plain area (root mean square of height $\sigma<100 \mathrm{~m}$ ) , general mountain area (root mean square of height $100 \mathrm{~m} \leq \sigma<200 \mathrm{~m}$ ) and mountain area (root mean square of height $\sigma \geq 200 \mathrm{~m}$ )[15] shown as table 1. Each kind of terrain have 12 triangles, so each group have 36 triangles ,and there are 72 triangles in all.

In this paper, we use two kind of methods for the simulation experiments performed, (a) given observations of gravity anomaly ( simulated by EGM2008), use the formula (10) and (19) directly for the estimation of unknown value of point $\mathrm{P}$. (b) using the remove-compute-restore technique. First ,we calculate the model gravity anomaly of the given points ( simulated by EGM2008) and the unknown point using high order gravity model (EGM96[16]) . Second ,calculate the residual gravity anomaly of the given points according to the given gravity anomalies and the calculated model gravity anomaly from formula (21). And then using the solution result we can estimate the residual gravity anomaly of unknown point from formula (17) and (22). And then use the residual gravity anomaly and the model gravity anomaly of the unknown point to recover the gravity anomalies of unknown point from formula(11). As for the noise, we also divided each experiment group into two categories, one category is supposed that noise covariance $D$ equal zero, and the another class is with the formula (24) depicted $D$.

\subsection{Experience Analysis}

The results are shown in Table $\mathbf{2}$, from the results we can see that :
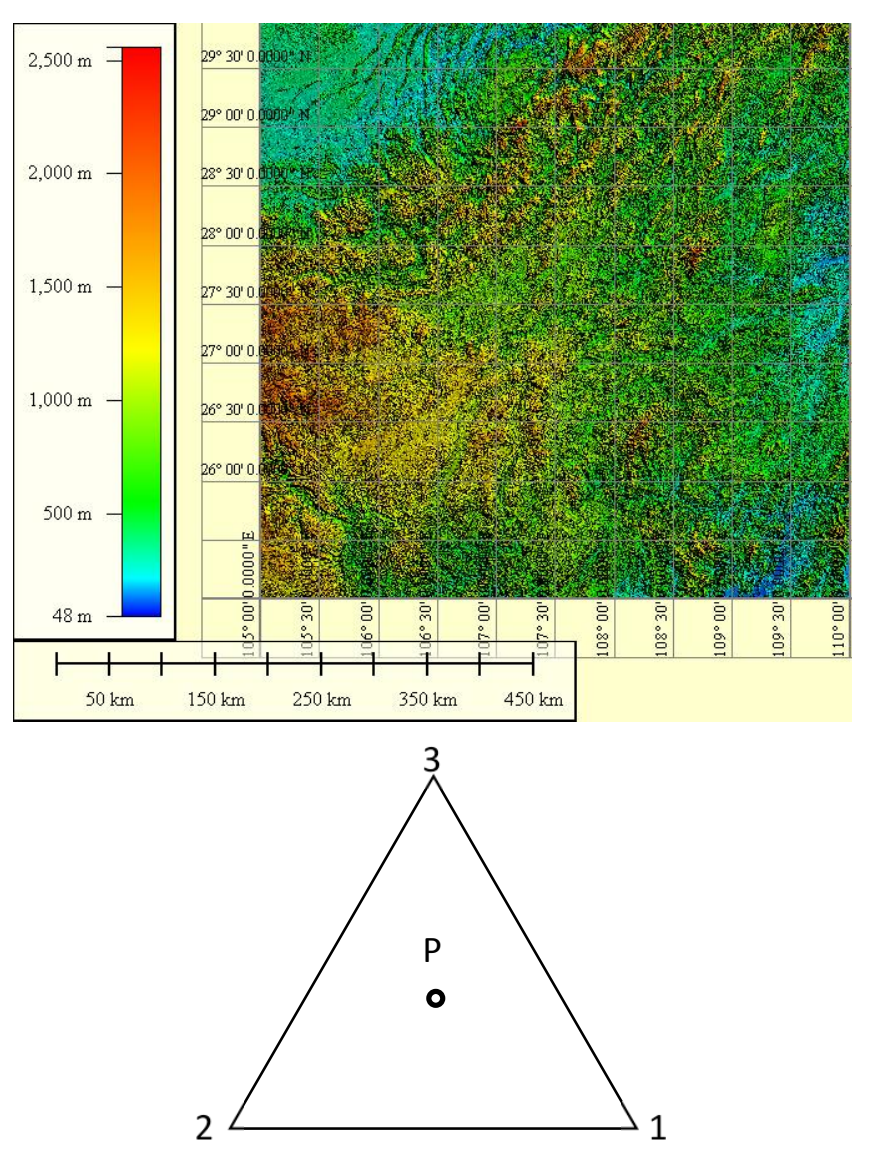

Fig. (1). The range of experience zone Fig. (2). The triangular of observation.

(1) Compared with the two groups of experiments of nonnoise covariance correction, we can see that with the increase of the given side length the estimation precision will reduced ,and the decreased rate almost equal to the increasing times of the given side length. This shows that there are some correlation between the accuracy of the estimation results and the given side length, the more long the given side length the more low the precision of the building covariance matrix.

(2) Compared with the two groups of experiments of including noise covariance correction, we can see that there is a certain correlation between the accuracy of the estimation results and the RMS of local terrain elevation from the simulation experience, the results are consistent with the noise matrix. In the condition of this noise matrix, with the increase of the given side length, the

Table 1. The results statistics (unit:m).

\begin{tabular}{|c|c|c|c|}
\hline & Plain area & General mountain area & Mountain area \\
\hline \hline Average Height (each edge length $\mathbf{1 5} \mathbf{~ k m}$ ) & 387.409 & 938.013 & 1463.873 \\
\hline RMS of elevation (each edge length $\mathbf{1 5} \mathbf{~ k m}$ ) & 44.079 & 161.754 & 309.606 \\
\hline Average Height (each edge length $\mathbf{3 0} \mathbf{~ k m}$ ) & 348.707 & 702.364 & 1233.800 \\
\hline RMS of elevation (each edge length 30 $\mathbf{~ k m})$ & 40.088 & 174.656 & 205.981 \\
\hline
\end{tabular}


Table 2. The standard deviation results statistics.

\begin{tabular}{|c|c|c|c|c|c|}
\hline $\begin{array}{c}\text { The edge length of given } \\
\text { points }\end{array}$ & & Method & Plain area & General mountain area & Mountain area \\
\hline \multirow{4}{*}{$15 \mathrm{~km}$} & \multirow{2}{*}{$\begin{array}{l}\text { Including noise } \\
\text { covariance }\end{array}$} & Directly compute & 3.448 & 4.231 & 15.557 \\
\hline & & Remove-compute-restore & 3.094 & 4.816 & 12.116 \\
\hline & \multirow{2}{*}{$\begin{array}{l}\text { None-including noise } \\
\text { covariance }\end{array}$} & Directly compute & 2.834 & 5.654 & 6.996 \\
\hline & & Remove-compute-restore & 2.838 & 5.897 & 7.027 \\
\hline \multirow{4}{*}{$30 \mathrm{~km}$} & \multirow{2}{*}{$\begin{array}{l}\text { Including noise } \\
\text { covariance }\end{array}$} & Directly compute & 3.696 & 18.765 & 14.111 \\
\hline & & Remove-compute-restore & 3.894 & 19.024 & 12.709 \\
\hline & \multirow{2}{*}{$\begin{array}{l}\text { None-including noise } \\
\text { covariance }\end{array}$} & Directly compute & 3.608 & 14.755 & 11.977 \\
\hline & & Remove-compute-restore & 3.985 & 14.656 & 11.55 \\
\hline
\end{tabular}

estimation accuracy of unknown point will change little in the same region. From the two set of experiments we can see that the calculation results acquired from removecompute-restore technique is better than that of directly calculation method.

(3)From the two groups of experiment results, the noise matrix correction reduces the accuracy of the estimation value. Therefore, the noise matrix correction still need further study, but because it is just the simulation experiment, the experimental data and the selection of composition and other factors will affect the experimental results.

\section{CONCLUSION}

In this paper, we proposed a new method to build covariance matrix of gravity anomaly based on the spherical harmonic expansion of gravity anomaly, at the same time ,we verified the feasible of building covariance of gravity anomaly using EGM2008 and then estimate the unknown point through the least squares collocation. With the development of ultra high-order gravitational model, it provides a new way of building the gravity anomaly covariance for us. This method combines the removecompute-restore technique will further improve the accuracy of estimation. Separate the given value into model gravity anomaly and residual gravity anomaly and use the calculated residual gravity anomaly to estimate residual gravity anomaly of unknown point, and then the result add the model gravity anomaly of unknown point to recover the solution of gravity anomaly of unknown point . In addition, the number effects of the given points and the noise covariance matrix effects of the given points for the results of recovery solution are still need to be further studied.

\section{CONFLICT OF INTEREST}

The author confirms that this article content has no conflict of interest.

\section{ACKNOWLEDGEMENTS}

This study is supported by the National Natural Science Foundation of China (No. 41374009), the Shandong Natural
Science Foundation of China (No. ZR2013DM009), the National Basic Research Program of China (No. 2013CB733302), the Public Benefit Scientific Research Project of China (No. 201412001), and the SDUST Research Fund (No. 2014TDJH101).

\section{REFERENCES}

[1] P. Knudsen, "Estimation and modeling of the local empirical covariance function using gravity and satellite altimeter data", Bulletin Geodesique, vol. 61, pp.145-160,1987.

[2] K. Christopher, "Least-squares collocation with covariancematching constraints", Journal of Geodesy, vol. 81 , pp. 661-667, 2007.

[3] D. N. Arabelos, and C. C. Tscherning, "Error-covariance of the estimates of spherical harmonic coefficients computed by LSC, using second-order radial derivative functional associated with realistic GOCE orbits", Journal of Geodesy, vol. 83, pp. 419-430, 2009.

[4] X. C. Zou, "The application of least-squares collocation in physical geodesy", MS thesis, Wu Han university, Wuhan, ON, China, 2003.

[5] W. A. Heiskanen, and H. Moritz, Physical Geodesy. W.H. Free man and Company, 1967, pp.174-199.

[6] H. Moritz, Advanced Physical Geodesy. Abacus Press, England, 1980.

[7] Z. Xia, and L. Li, "Approximations of local covariance functions of Gravity Anomaly", Acta Geodaetica et Cartographica Sinica, vol. 24, no. 1, pp.23-27, 1995.

[8] N. K. Pavlis, S. A. Holmes, S. Kenyon, and J. K. Factor, "The development and evaluation of the Earth Gravitational Model 2008 (EGM2008)", Journal of Geophysical Research, vol. 117, B04406. 2012, doi:10.1029/2011JB008916.

[9] C. Y. Zhang, C. X. Guo, and J. Y. Chen, "EGM2008 and its application analysis in Chinese mainland", Acta Geodaetica et Cartographica Sinica, vol. 38, no. 4, pp. 283-289, 2009.

[10] C. Li, Y. M. Wei, Z. K. Liu, and L. Y. Ji, "Calculating the elevation abnormality by the method of RCR based on CQG2000", Hydrographic Surveying and Charting, vol. 27, no. 4, pp. 31-33, 2007.

[11] X. Ju, "Regional research on the merging between land geoid and ocean geoid", MS thesis, Shandong University of Science and Technology, Qingdao, ON, China, 2011, pp. 27-40.

[12] X. G. Liu, Y. Deng, X. S. Ye, and S. Wu, "Precision comparison of the earth gravity field models of EGM96 and EGM2008", Hydrographic Surveying and Charting, vol. 30, no. 2, pp. 55-57, 2010.

[13] R. Forsberg, and C. C. Tscherning, "The use of height data in gravity field approximation by collocation", Journal of Geophysical Research, vol. 86, pp. 7843-7854,1981. 
[14] W. He, and L. Lou, "Regional geoid determination methods based on the remove-restore technique", GNSS World of China, vol. 6, pp. 32-37, 2009.

[15] P. Meissl, "Preparations for the Numerical Evaluation of Second Order Molodenskey-type Formulas , Rep. 163", Dep. of Geod. Sci. , Ohio State University , 1971
[16] F. G. Lemoine, S. C. Kenyon, R. G. Factor, R. G. Trimmer, N. K. Pavlis, D. S. Chinn, C. M. Cox, S. M. Klosko, S. B. Luthcke, M. H. Torrence, Y. M. Wang, R. G. Williamson, E. C. Pavlis, R. H. Rapp, and T. R. Olson, "The development of the joint NASA GSFC and the National Imagery and Mapping Agency (NIMA) geopotential model EGM96", NASA/TP-1998-206861 Goddard Space Flight Center, Greenbelt, USA, pp.575, 1998.

(C) Wang et al.; Licensee Bentham Open.

This is an open access article licensed under the terms of the (https://creativecommons.org/licenses/by/4.0/legalcode), which permits unrestricted, noncommercial use, distribution and reproduction in any medium, provided the work is properly cited. 